\title{
BIOMED concerted action chemical sensors for in vivo monitoring. The biocompatibility issue
}

\section{First Workshop held in Fontainebleau, France, June 7-9, 1993.}

\author{
G. Reach $^{*}$
}

INSERM U341, Diabetes Department, Hotel-Dieu, 1, Place du Parvis

Notre Dame, 75004, Paris, France.

\section{J. Feijen,}

Department of Chemical Technology, University of Twente, PO Box 217, 7500 AE Enschede, The Netherlands

\author{
S. Alcock \\ Cranfield Biotechnology Centre, Cranfield University, Cranfield, \\ Bedford MK43 OAL, UK. \\ *Author to whom all correspondence should be addressed.
}

What are the problems with in vivo use of sensors in terms of surface materials, device morphology and charge, infection and toxicology? Will the body's responses significantly affect the responses of the device?

In 1989, a Biomedical Engineering (COMAC-BME) concerted action of the European Community involving a network of 29 laboratories in the field of continuous monitoring was founded under the name of "Chemical sensors for in vivo monitoring". During the three year period of this action, two annual meetings were held, between-labs exchanges were granted, a newsletter was founded, and two books were edited (Alcock \& Turner, 1993; Turner, 1993). In 1993, the action, now involving 37 labs, was renewed under the framework of Biomed 1. This report describes the papers presented during the first workshop of this renewed action, held in Fontainebleau, June 7-9, 1993, entitled: The Biocompatibility Issue.
A chemical sensor can be defined as a device able to monitor continuously the concentration of an analyte, specifically recognized by a detection element (an enzyme, an antibody, a receptor, a cell etc...), leading to a chemical reaction which is transduced into a signal that can be processed to yield an estimation of the analyte concentration. Obviously, such systems bear a tremendous potential for applications in several fields of medicine. However, strategies for development have to be defined, taking into account both the specific clinical application itself, the current state of the art of the available technologies, and the reaction of the host, which will defend itself against the implant. 
If one considers glucose monitoring as a paradigm of continuous in vivo monitoring (G. Reach, Paris, France), glucose sensors have been incorporated in a bedside artificial pancreas able to correct hyperglycaemia over a few days. A glucose sensor may also be useful for educational purpose to demonstrate to diabetic patients the characteristics of their blood glucose profile. If however, one wants to use a glucose sensor as a tool for the daily treatment of diabetes mellitus on a long term basis, it becomes important to consider the longevity of the sensor. If the sensor is supposed to be implanted, it is essential to control the possible adverse effects of the environmental tissue in which the sensor is implanted, which may alter the sensor's function, and on the other hand, the effects of the sensor on the tissue in which it is implanted (such as inflammation or infection, coagulation if the sensor is implanted in a vascular bed etc...). Thus the biocompatibility issue appears as a major topic in the field of sensor development. Understanding the phenomena and the mechanisms which are behind this word is therefore critical, explaining the choice of the biocompatibility issue as the topic of the first workshop of the renewed Concerted Action.

\section{Surface materials}

Materials are an inevitable consideration (P. Vadgama, Manchester, UK) in the design of chemical biosensors, even with regard to basic transduction mechanisms and operation in simple solution. Thus, whether a carbon, a noble metal, or indeed a chemically modified working electrode is used in an amperometric device, a prior assessment of relevant materials properties is implicit in the choice. This is equally so with incorporation of a bioreagent in biosensors, since the incorporation of reagent such as an antibody or enzyme into the solid phase amounts to the creation of a new type of material with distinct bulk phase and surface properties. However, in most instances, experience is that of the chemical functionality of such entities in controlled solution environments where there is some predictability over behaviour. When a sensor is exposed to the in vivo blood or tissue environment, quantitative prediction of performance, particularly as regards stability, becomes difficult. Materials considerations here acquire a new relevance and dimension, and aspects other than intrinsic chemical functionality require careful evaluation and subsequent optimisation.

A key material component of the sensor is the outer covering layer used for the analyte recognition process. A defined strategy for limiting the deposition and growth on this layer of surface-active colloidal species, notably proteins, lipids and cells, becomes central to useful sensor function. Relevant material properties influencing surface deposition processes include roughness, charge, hydrophilicity, chemical functionality and crystallinity. It is also necessary for the materials to have the desired solute (analyte) permeability properties, as well as a rejection capability for interferent species. One convenient strategy is to utilise polymeric covering and barrier layers within existing devices. These strategies for "packaging" also have the potential to limit leaching of potentially toxic electrode components and can mask antigenic components.

An approach to optimisation has involved the development of a multiple polymeric membrane enzyme laminate needle electrode for in vivo implantation (P. Vadgama, Manchester, UK). In particular, surface haemocompatibility has been enhanced by an outer polyarylsulphone dip coated membrane. Inner permselective polyurethane membranes have provided effective exclusion of naturally occurring electrochemically active interferents in blood from reaching the working electrode. The absence of such a membrane causes spurious non-predictable elevation of responses, rendering such sensors unreliable.

A further material, Diamond Like Carbon (DLC), which forms strong yet flexible amorphous coatings has shown to impart hitherto unforseen levels of biocompatibility in vitro when used to coat low porosity outer covering microporous polycarbonate membranes in an enzyme laminate electrode. Furthermore, DLC coatings were found to offer novel permselective properties, effectively screening the underlying 
sensor from naturally occurring interferents within whole blood, so permitting the determination of blood glucose levels without the use of an underlying permselective membrane. It therefore follows that DLC may lend itself to the future coating and thereby optimisation of invasive in vivo sensors.

During the last decades several groups have been active in the design of blood compatible polymers and polymer surfaces to be used in medical devices and artificial organs (J. Feijen, Twente, The Netherlands). When a polymer surface is exposed to blood several processes will take place, which often lead to thrombus formation. These processes include protein adsorption, conformational changes of proteins, activation and cellular adhesion and activation. The processes are interrelated and of a complex nature. Several approaches have been followed to control, prevent or suppress these processes.

The first approach is directed to the preparation of polymer surfaces which have a minimal interfacial free energy with blood. Surfaces have been modified with poly (ethylene oxide) (PEO), either by incorporation of PEO blocks in the main chain of the polymer or by grafting PEO onto the surface. Another method is the treatment of surfaces with a gas plasma to render the surface hydrophilic. A second strategy is the attachment of aliphatic chains to the surface, to establish a preferential adsorption of albumin. It has been found that albuminated surfaces suppress both the activation of the intrinsic coagulation and the adhesion of platelets. A third approach is the development of phase separated surfaces, having both hydrophilic and hydrophobic domains. In this way preferential protein adsorption can take place and conformational changes of adsorbed proteins may be minimized. This leads to a decreased platelet adhesion and activation.

Another direction is the attachment of the anticoagulant heparin to polymer surfaces. Heparin interferes with the formation of fibrin and when attached in the appropriate way it also reduces platelet adhesion and activation. Other approaches include the coupling of urokinase (fibrinolytic action), prostaglandin (prevention of the activation of platelets) and the seeding of endothelial cells. In designing blood compatible devices flow conditions should be taken into account. In spite of these difficulties, intravascular sensors can be used now with profit especially in critically ill patients, in whom it is important to monitor continuously several parameters, such as blood gas. The biocompatibility issue is an essential consideration for the long term use of a sensor, which may be jeopardized by events such as clotting or infection leading to unacceptable sensor drift, or sudden loss of the signal (P. Rolfe, Keele, UK).

It is essential to consider carefully the structure and properties of the sensor outer membrane, which is in direct contact with the host tissues (H. Strathmann, Twente, The Netherlands). The role of this membrane is to provide high transport selectivity and high transport rate for analytes to be measured, and to avoid leakage of potentially toxic components from the sensor. It must be chemically and mechanically stable, and "biocompatible". Different kinds of membranes (porous structures, homogeneous solid or liquid films, composite structures, asymmetric membranes) have been developed for other applications, and may not be suitable for the function of a glucose sensor. The development of membranes with specific transport properties such as multimatrix structures or materials containing functional groups may represent a clue for tailoring membranes appropriate for a given application.

It is difficult to describe the complexity of the networks involved in what is called the inflammatory reaction (N. Haeffner-Cavaillon, Paris, France): complement activation, macrophage activation leading to the production of multiple cytokines, the evolution towards chronic inflammation or resolution of inflammatory process, including angiogenesis and wound repairs, depends on the balance of proinflammatory/antiinflammatory cytokines: during the initiation of inflammatory reactions, attracted polymorphonuclear neutrophils and monocytes adhere to the protein layer adsorbed onto the artificial material and participate in the diverse effects of inflammation by producing 
locally a variety of cytokines. Biocompatibility will therefore depend first on protein adsorption (at least 200 proteins are known to circulate in plasma); attracted cells will produce cytokines (more than 50 of them are well characterized). Several cytokines and as yet incompletely characterized factors tightly regulate angiogenesis and wound repair which may finally allow tolerance of the material.

\section{Morphology and charge}

To investigate the possibility that morphological characteristics of implanted sensors might relate to the observation of a decrease in sensor sensitivity during implantation in human subcutaneous tissue, scanning electron microscopy (SEM) on explanted sensors was performed (J. Pickup, London, UK). The outer membrane was invariably very uneven, with coating defects acting as micro-pores, and presumably contributing to the permeability. The membranes were subject to inadvertent mechanical damage on some occasions, with stripping from the underlying enzyme layer. Although there was some cellular attachment to the sensors (red cells, fibroblasts), this was minimal, and after $8 \mathrm{~h}$ most of the deposition was an amorphous material, presumably protein and components of the extracellular matrix. Accumulation of both the cells and the amorphous material was considerably less in sensors which were poised at the set voltage $(700 \mathrm{mV})$ compared to those unpoised. Thus, impaired responses in vivo in the short term may not be due to cellular encapsulation, but protein and/or small molecular weight substances may be important. Charge may influence the biocompatibility of sensor membranes and care must be taken to avoid mechanical damage on insertion, during operation or on explantation of sensors.

Biomaterial insertion into tissue results also in phenomena such as protein adsorption to the material surface, followed by inflammatory responses (N. Ziats, Cleveland, USA). The inflammatory response (acute and chronic) and subsequent wound healing involve both surface interactions which are important for short term applications, and bulk material interactions which increase in importance as the intended in vivo use time increases. The use of stainless steel cages made it possible to investigate the host reaction to a subcutaneously implanted electrode: blood proteins, cells, and cellular derived materials collected on the metal surface of the electrode, which was associated with changes in the electrode impedance. It was suggested that this change may be related to the tight adherence of cells on the electrode.

\section{Surface evaluation}

The major problems related to the outer membrane interface of biosensor devices include fouling of these surfaces due to protein adsorption and cellular deposition and the presence of accumulated depleted reactive species. Since these phenomena are directly mediated by the morphology and chemical nature of membrane surfaces, their characterization is essential for yielding useful information on future development of biosensor systems (A. Baszkin, Chatenay-Malabry, France). The surface characterization methods related to these problems were reviewed with the emphasis on the following test procedures: combination of the Washburn and the Neumann equations which enables determination of the pore size from the wettability measurements; electron spectroscopy for chemical analysis (ESCA) for semiquantitative analysis of all elements present and information on the molecular bonding environment for most atoms in the surface; static secondary ion mass spectrometry (SIMS) which provides molecular information on the type of surface chemical species present and their possible structure; Fourier transform infrared spectroscopy combined with attenuated total reflection spectroscopy (ATR-FTIR) which is used to study molecular structure and surface morphology. Monitoring of protein adsorption in contact with a flowing biological liquid may be obtained; scanning electron, scanning tunnelling and atomic force microscopes enable imaging with different resolution degrees of adsorbed proteins and their structures. 


\section{Infection}

Another point to consider carefully for a long term implanted sensor is infection (J. Dankert, Amsterdam, The Netherlands). Adherence of bacteria to the surface of a foreign body is considered to be the first step in the pathogenesis of foreign body-related infections. Bacterial attachment to the foreign body is a function of the surface area, the hydrophobicity, the porosity and smoothness of the foreign body: increased bacterial adherence occurs on large surface areas with increased surface hydrophobicity and on a rough surface. The surface characteristics of biomaterials in vitro are different from those of the biomaterial in vivo, since host proteins and cells coat the implanted biomaterial. Staphylococci possess properties to bind selectively to certain proteins and to deposited cells. The binding of bacteria to the biofilm present at the surface of the foreign body is complex and depends on a number of specific interactions between the bacteria and the proteins, and the conformation of these proteins. In addition, in vivo, various macromolecules will bind onto the bacterial surface, leading to a change of the bacterial cell surface properties relevant in the adhesion process.

\section{Toxicity}

It is of importance to establish whether sensors implanted in vivo possess toxic properties. The toxic properties could be derived from the sensor itself or, in the case of enzymatic sensors, from the products of the enzymatic reaction. Most enzymatic sensors possess thin polymer membranes which are deposited from organic solvent solutions. Residual solvent trapped in the polymer matrix can leach out into the surrounding tissue. Other reagents such as glutaraldehyde, the enzyme or other proteins (e.g. BSA) added to the sensing element must also be considered. If a reference electrode such as $\mathrm{AgCl} / \mathrm{Ag}$ is present, then the effects of $\mathrm{Ag}$ should be evaluated. It is also desirable to spatially resolve the source of toxicity. Is it coming from the sensing element, the reference electrode, or some other part of the sensor?
A method for cell culture toxicity testing, involving the use of a mouse fibroblast cell line, was presented (G.S. Wilson, Lawrence, USA). A modification of the Agar Diffusion method made it possible to study intact sensors directly and to distinguish toxicity evolving from the sensing element and that of the reference electrode. The major conclusion was that residual solvent in the polymer layer (primarily tetrahydrofuran /dimethylformamide) and possibly the crosslinking agent glutaraldehyde are the major toxic substances. However, if the sensor is equilibrated with buffer for 5 days the toxicity disappears. This coincides with the stabilization of outer membrane transport properties. It is also necessary to consider other localized effects resulting from the production of products of the enzymatic reaction such as peroxide or possible immunological effects of glucose oxidase.

\section{Transport}

Is it possible to model the effect of the fibrotic reaction to the implanted sensor, witness of an inappropriate biocompatibility, on the function of the sensor (K. Dionne, Providence, USA)? Steady state and transient responses of implanted enzymatic glucose sensors depend on consistent electrode performance and on predictable transport resistance of glucose and co-factors, such as electron accepting oxygen, from the bulk tissue to the electrode surface. The development of a fibrotic reaction between the sensor and the bulk tissue in response to membrane bioincompatibility may affect sensor performance by any of three pathways: 1) increased transport resistance can delay the transient response of the sensor to changes in glucose concentration in the bulk tissue; 2) glucose consumption by metabolically active fibroblasts can alter the relationship between the glucose concentration immediately surrounding the sensor and bulk tissue or blood glucose concentration; 3 ) both metabolic consumption and increased transport resistance of the fibrotic layer to co-factors may deplete factors such as oxygen to such an extent that cofactor availability or fluctuations in concentration become the rate determining step in 
sensor output. Each of these mechanisms can be mathematically incorporated into sensor algorithms assuming i) the fibrotic reaction reaches a steady state, ii) the extent of fibrosis is not such that performance characteristics become unacceptablc, and iii) the probe can be recalibrated in vivo.

Sensor time lag due to glucose transport scales to the square of the thickness of a nonvascularized fibrotic layer. Roughly a $0.1 \mathrm{~mm}$ fibrotic overgrowth will add $0.5 \mathrm{~min}$ time lag to the characteristic transient sensor response time whereas a $1.0 \mathrm{~mm}$ overgrowth will add approximately $50 \mathrm{~min}$. Depletion of the glucose or oxygen concentration at the sensor as a result of metabolic consumption and transport resistance of the boundary layer is a function of reaction thickness, metabolic rate, effective mass transport coefficient, and electrode geometry. Metabolic reductions will initially increase, and then slowly decrease as collagen replaces metabolically active fibroblasts as the primary component in the reaction layer. Reaction/diffusion reaction models suggest that in most configurations, glucose reduction due to glucose metabolism will not be significant where the fibrotic reaction is less than $1 \mathrm{~mm}$ thick. Oxygen tends to be more constrained than glucose, however several groups have developed sensor designs wherein oxygen is either not required or is not rate limiting.

\section{Experimental results}

Enzymatic electrodes were implanted in the subcutaneous tissue of diabetic dogs (U. Fischer, Karlsburg, Germany): stable glucose-related sensor signals were observed over periods of 14-168 $\mathrm{h}$. The loss in sensor function (appearance of non-glucose related oscillations and/or loss in sensitivity) was accompanied by acute inflammation which was analyzed by means of conventional histology. The observations apply to subcutaneous implantation of amperometric glucose sensors which were neither strictly sterile nor appreciably miniaturized. Under these conditions, the host response to the sensor provides the gross mechanism of loss in sensor function which may simply be caused by the diffusion barrier and the low glucose concentration of peri-sensor exudative fluid. This may be ameliorated by miniaturization and sterilization of sensors but it appears also worthwhile to investigate the immune mechanisms of host response to sensor biomaterials and to circumvent the necessity of permanent skin penetration by sensor wiring.

In vivo testing of glucose sensors implanted subcutaneously in normal rats was performed using different glycaemia variation patterns and implantation times (M. Koudelka, Neuchatel, Switzerland). The sterilized sensors were implanted in the interscapular region under a pentobarbital anaesthesia. Following a baseline period of $90 \mathrm{~min}$ to reach a reasonably stable current and the in vivo calibration, the correlation between the plasma glycaemia and the apparent subcutaneous glucose levels was investigated during controlled glucose and/or insulin infusions and following intraperitoneal glucose loads or intramuscular glucagon-insulin administrations. Sensor function was demonstrated for three days following implantation in all cases. Local anaesthesia with lidocaine proved to have an adverse effect on sensor function.

One study examined whether an immune response against the glucose biosensor membrane is induced after implantation, and when so, what kind of membrane material is distinguished by lowest immunogenicity (M. Ziegler, Karlsburg, Germany). Sterilized pieces of membranes (10 $\mathrm{mm}^{2}$ ) consisting of polyimide, polyurethane, polycarbonate or teflon were subcutaneously implanted into rats. The implantation was repeated at experimental days 21 and 42 . In parallel the first implantation was carried out in combination with an intraperitoneal injection of complete Freund's adjuvant. The results suggested an unexpected antibody formation against membranes used for the glucose sensor, and that only polyimide seems to be immunologically inert.

Electroenzymatic glucose sensors implanted into subcutaneous tissue of human subjects and experimental animals (W. Kerner, Ulm, Germany) exhibited lower sensitivities to glucose 
than in buffer solutions before implantation. Sensors were inhibited in human plasma or plasma ultrafiltrate (mol.wt. $<10 \mathrm{kDa}$ ). Losses of sensor sensitivities to glucose in subcutaneous tissue and in plasma were totally reversible upon re-exposure of sensors to buffer solutions. It may be therefore that sensor inactivation in plasma and possibly in subcutaneous tissue is caused by low molecular weight substances not retained by the polyurethane membrane of the glucose sensor.

\section{Clinical trials}

Results of a clinical evaluation of miniaturized sensors implanted for $18 \mathrm{~h}$ in the subcutaneous tissue of human volunteers was presented (G. Reach, Paris, France). After cleaning of the skin with betadine, the sensor was implanted over $1 \mathrm{~cm}$ through a 21 gauge needle, which was then removed, leaving the sensor in place. No anaesthesia was required. A minimal, transient, inflammatory reaction (1-2 cm diameter red, painless area surrounding the sensor implantation site) was observed in 8 cases (54 trials). There was no relation with the function of the sensor, which was assessed by monitoring the current during an oral glucose tolerance tests. Such a reaction was observed in only one out of 7 subjects in which the sensor was implanted for 4 days. It may be important to understand the cause of this reaction, even though it was minimal and inconstant. Possible causes include the leakage of some component from the sensor, the presence of pyrogens, or an inadequate biocompatibility of the outer polyurethane membrane.

Experience with a bedside prototype artificial pancreas using an enzyme thermistor for glucose measurement in whole blood was presented (B. Danielsson, Lund, Sweden). Blood is sampled through a double lumen catheter suitable for local blood anticoagulation. At this site the biocompatibility issue was encountered.

\section{Microdialysis probes}

A microdialysis probe realized with a hollow fibre was coupled with a wall jet electrochemical cell provided with a glucose sensor (M. Mascini, Firenze, Italy). The probe was implanted in rats, rabbits and recently in human beings. In the latter case, the probe was sterilized with cthylene oxide. The examination period was generally of 3 hours, during which an oral glucose tolerance test was performed. Muscle contraction, in a few cases, caused flow variation and resulted in a complex response. Smoothing procedures were necessary to eliminate the rapid variation due to contraction.

By perfusing a hollow fibre microdialysis probe inserted into subcutaneous fatty tissue, a reliable sampling method for on-line glucose measurements could be achieved in the experience of A.J.M. Schoonen \& F. Schmidt (Groningen, The Netherlands). The reproducibility of the microdialysis system allows direct interpretation of the in vivo sensor signal after prior in vitro calibration. This is surely a main advantage of the system. However, perfusion of enzyme solution through the hollow fibre probe revealed a possible dangerous side-effect: the enzyme could enter the body. This results in a swelling and reddening of the skin that may be painful. In the subcutaneous tissue the enzyme continues to react with glucose, producing hydrogen peroxide. Furthermore sensitization to the enzyme occurs after repeated exposure.

The chance for glucose oxidase, being allergenic, to enter the body needs to be excluded before safe in vivo use of the glucose sensing device can be guaranteed. To solve this safety problem the subcutaneous microdialysis system was redesigned such that the glucose within the perfusion fluid was only allowed to react with the enzyme outside the body. This can be realised by introducing a glucose oxidase reactor, based on passive diffusion, between the dialysis probe and the final oxygen detector. Small traces of enzyme that might escape from the reactor chamber are captured by a combined active coal/filtration system before the perfusion fluid can re-enter the microdialysis probe circulation. Complete prevention of enzyme leakage can thus be guaranteed without sacrificing the principle of the closed-loop microdialysis system, allowing long-term ambulatory studies with the glucose sensor. 


\section{Free communications}

A free communication session included presentations on biocompatibility and regeneration issues in relation to ex vivo TIRF immunosensors (C. Domenici, Pisa, Italy) and use of heparin to modify the surface of a cuprophan membrane and improve biocompatibility (G. Engbers, Twente, The Netherlands). It concluded with an on-line demonstration (S. Alcock, Cranfield, UK) of the Concerted Action database on chemical sensors for in vivo monitoring, which can be accessed and searched directly from remote sites in Europe (Alcock and Knight, 1993).

\section{Regulatory issues}

This workshop was concluded by the presentation of a matrix for evaluation guidelines which are currently proposed in Europe as the ISO recommendations (ISO 10993) for the Evaluation of Biocompatibility of Biomaterials (M.R. Eloy, Chasse sur Rhone, France). The risk and testing procedures are related to the site and duration of implantation of the material or devices. These guidelines are precise on the method to be used for test selection, animal welfare requirements, tests for genotoxicity, carcinogenicity, and reproductive toxicity, the selection of tests for interaction with blood, in vitro methods for in vitro cytotoxicity, lests for local effects after implantation, tests for screening sterilization residues, the degradation of materials related to biological testing, tests for irritation and sensitization, and tests for systemic toxicity.

\section{Conclusion}

Biocompatibility of a material can be defined by the consequences of the events occurring at the interface with the host tissue or blood in which it is implanted (G. Reach and J. Feijen),. It is essential to understand that this definition is highly relative, and that the same "event" can prove to be either beneficial or harmful, according to the application for which the material is supposed to be used. The host reaction can indeed be harmful (effect of low molecular weight components coming from the milieu interieur and altering the sensor's characteristics, fibrosis preventing the access of the analyte to the sensor for instance) or beneficial (angiogenesis facilitating this access). Can it be controlled? On the other hand, the sensor versus host reaction can also have local or systemic harmful effects: infection, chronic inflammation; it must be evaluated according to regulatory rules. Another point to consider in the case of a disposable sensor is the repetition of implantation.

Finally, three other messages can be outlined from this workshop:

1. There is no inert implant, since an implant...has to be implanted

2. Consider not only the biomaterial present at the outer surface of the sensor, but all biomaterials used in its design

3. Biomaterials can be characterized, and even engineered in the framework of a rationalized tailoring process.

Clearly, in the biosensor field, biocompatibility appears now as a fascinating and still open, but certainly essential, issue.

\section{REFERENCES}

Alcock, S.J. \& Knight, C.M. (1993). Database on chemical sensors for in vivo monitoring. In: Technology and Health Conference Issue. Elsevier, The Netherlands, 304-305.

Alcock, S.J. \& Turner, A.P.F. (eds) (1993). In: In vivo chemical sensors: recent developments. Cranfield Press, Cranfield (UK), 199 pp.

Turner, A.P.F. (ed). (1993). Advances in Biosensors Supplement 1: Chemical sensors for in vivo monitoring. JAI Press, London (UK), 258 pp.

\section{FURTHER INFORMATION}

Further information on the Concerted Action can be obtained from the Project Leader, Professor A.P.F. Turner, Cranfield University, Cranfield, Bedford, MK43 OAL, UK. 